\title{
Design of Remote Structural Monitoring System Based on Labview and Linux
}

\author{
Yubing Jing*1, a , Hui Zhao ${ }^{2, \text { b }}$ \\ ${ }^{1}$ Yubing Jing, China \\ ${ }^{2}$ Hui Zhao, China \\ ${ }^{1,2}$ Zhonghuan information college, Tianjin University of technology, Tianjin 300380, China \\ aemail: jingyubing@163.com, bemail: tj_zhaohui@163.com
}

\begin{abstract}
In order to keep the safety of the large structures, it is necessary to take effective remote monitoring for it. The design method of a remote structural monitoring system based on Labview and Linux is proposed in this paper, and the constitution modulus and function realization of the system are presented too. In the system, the network transmission of field data, real-time data display, monitoring, the calculation of structural modal parameters and other functions has been realized, and the intranet constructed by monitoring points is also realized through embedded technology.
\end{abstract}

Keywords- remote structural monitoring; labview; linux.

\section{INTRODUCTION}

In recent years, people pay more and more attention to the safety and durability of large structure buildings, and remote structural monitoring has also become a hot topic in academic and engineering circles. Remote structural monitoring, that is using nondestructive sensing technology and modern network technology, through the analysis of structure system characteristics including structural response, to realize the purpose of detecting structural damage or deterioration.

Following, a remote monitoring system for large structures has been designed and developed, monitoring content mainly includes a variety of physical quantities involved the monitoring point, for example, temperature, strain, deflection, velocity, acceleration, and so on. The modal parameters of the structure have been calculated by its response parameters, as the basis for analyzing the structural state evaluation.

\section{THE COMPOSITION OF REMOTE STRUCTURAL MONITORING SYSTEM}

The remote structural monitoring systemis the automatic system of online monitoring under the minimal manual intervention ${ }^{[1]}$. It can automatically report structure state via LAN (Local Area Network) or remote center, so as to realize the online monitoring and controlling.

The constitution diagram of remote structural monitoring system is shown in Fig.1, which consists of three parts: client, monitoring center, and monitoring points. Monitoring center uses Internet as the data transmission channel, and through B/S (Browser/Server) mode, makes any client can realize software management operation.
Monitoring center has realized real-time data acquisition, online display, early warning, data query, and calculating structural fingerprint parameters etc. In this system, the monitoring points use ARM development boards, and they are mainly responsible for the acquisition and transmission of network data. Multiple monitoring points constitute an intranet, the communication between monitoring point and monitoring center can use the wired network or wireless network in two ways, and the development of the wired network communication mode has been completed.

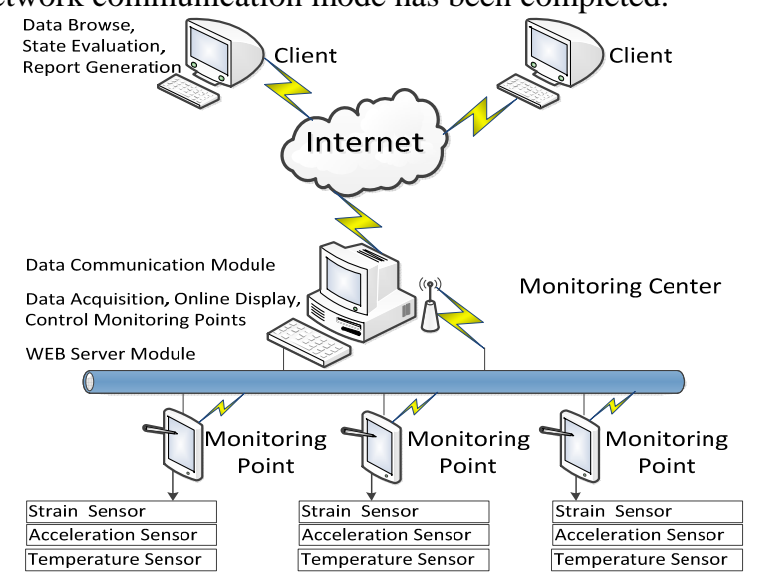

Figure 1. The constitution diagram of remote structural monitoring system

The system has good expansibility. The monitoring center could be extended to multilevel monitoring system through the Ethernet technology. This mode not only scatters the working pressure of the central station system, but also disperses the failure risk, and can make the whole system work more stable. Because of the maturity of the Internet technology, it is greatly reducing the cost of investment. The Monitoring point processor uses Samsung Corp's S3C2410 chip with high data processing ability. The monitoring points can also be extended to multilevel acquisition system, this make full use of the data processing ability of slave computer, alleviate the pressure of large quantities data of monitoring center.

\section{THE REALIZATION OF THE SYSTEM FUNCTIONS}

According to the features of software module and the advantages of different development platform, the remote structural monitoring system chooses the development 
mode of multi-platform. Because of the outstanding advantage of Labview in virtual instrument technology and network communication technology ${ }^{[2]}$, the remote structural monitoring system adopts Labview as the main developing platform.

\section{A. The design of monitoring center}

Software module structure diagram of monitoring center is shown in fig.2. Can be seen from the graph, monitoring center consists of two parts: communication module and WEB Server module. Communication module includes: sampling and communication submodule, online display submodule, early warning system submodule. WEB Server module includes: data report submodule, data query submodule, safety evaluation submodule, damage diagnosis submodule. Each module through the interface configuration between different platforms can be automatically invoked running. It makes full use of the advantage of software resource, and improves the accuracy and efficiency of the system.

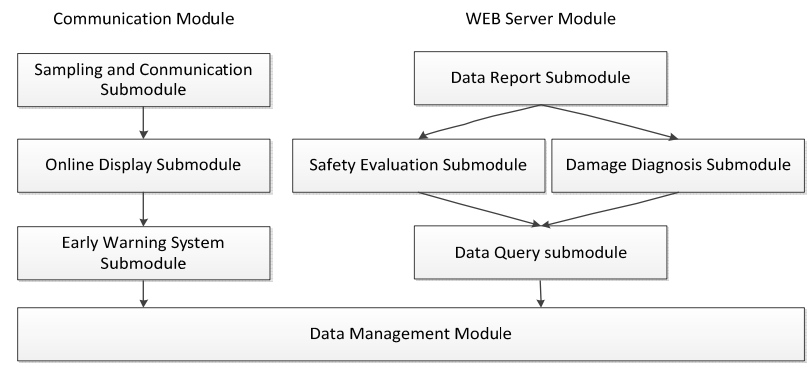

Figure 2. Software module structure diagram of monitoring center

\section{1) Communication submodule}

There are two kinds of networking scheme about the data communication between monitoring center and monitoring points, one is to use Ethernet wired communication mode, one is to use GPRS (General Packet Radio Service) wireless communication mode. In the Ethernet wired communication mode, because of the problems of NAT (Network Address Translation) mapping between public IP (Internet Protocol) and APN (Access Point Name) network IP, the monitoring center could not active addressing the monitoring point, only the monitoring point active connect to monitoring center. GPRS wireless communication mode can realize the bilateral control of monitoring center.

The communication data packet protocol between monitoring center and monitoring point is shown in table I.

TABLE I. COMMUNICATION DATA PACKET PROTOCOL

\begin{tabular}{|c|c|c|}
\hline Packet format & Byte & Content \\
\hline ID & 1 & $55 \mathrm{H}$ \\
\hline VERSION & 1 & $0 \mathrm{x} 1$ \\
\hline destination address & 6 & The first four IP, after two hold \\
\hline source address & 6 & The first four IP, after two hold \\
\hline Packet type & 1 & \\
\hline Packet count & 1 & \\
\hline Packet length & 2 & \\
\hline Data & $*$ & 16 byte \\
\hline CRC & 2 & \\
\hline
\end{tabular}

The characteristics of remote structural monitoring system is multiple point dispersion, a tiny quantity of data, high real time, and more terminal numbers and so on, so the system selects UDP (User Datagram Protocol) protocol with high transmission efficiency. UDP is a connectionless communication protocol, which does not guarantee that data packets arrive at the destination site, so the application layer protocol of the system has joined acknowledgment and retransmission mechanism, it makes the whole system communication process more reliable. Data receiving flow of the communication submodule is shown in fig.3.

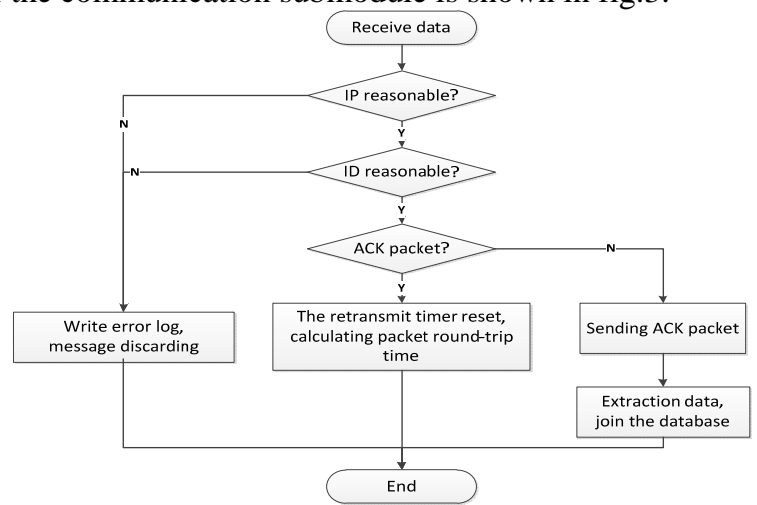

Figure 3. Data receiving flow of the communication submodule.

In order to ensure that the data accurately arrive at the receiving end, the application layer communication protocol of the system demand the receiving end to confirm for each of the received messages, if waiting longer than the delay time, the system will resend a message. If timeout three times, it will produce warning, and consider the terminal is unreachable. So choosing appropriate delay time can affect the real-time performance of the whole system. The system adopts self-adaption delay time value algorithm, it will be based on the actual message RTT (Round-Trip Time) to calculate each message RTO (Resend Time Out). The system also set up a RTT estimator:

2) Online display and early warning submodule

Online display and early warning submodule are developed under the Labview platform, modules have two kinds of running mode, one is the real-time monitoring network connection, receiving data and display, the other is according to the requirements of monitoring center to set the sampling frequency, the sampling points and other parameters of monitoring point. The flow diagram of data acquisition module is shown in fig.4. After the initialization module, set data acquisition mode of the system. If using the real-time acquisition mode, start monitoring port until the data arrives, then establish the UDP (User Datagram Protocol) connection, start using data receiving module. If using the control acquisition mode, start using the data sending module to send command word, and then receive the response, if the response is correct then began to establish the UDP connection, and to receive the data packet. 


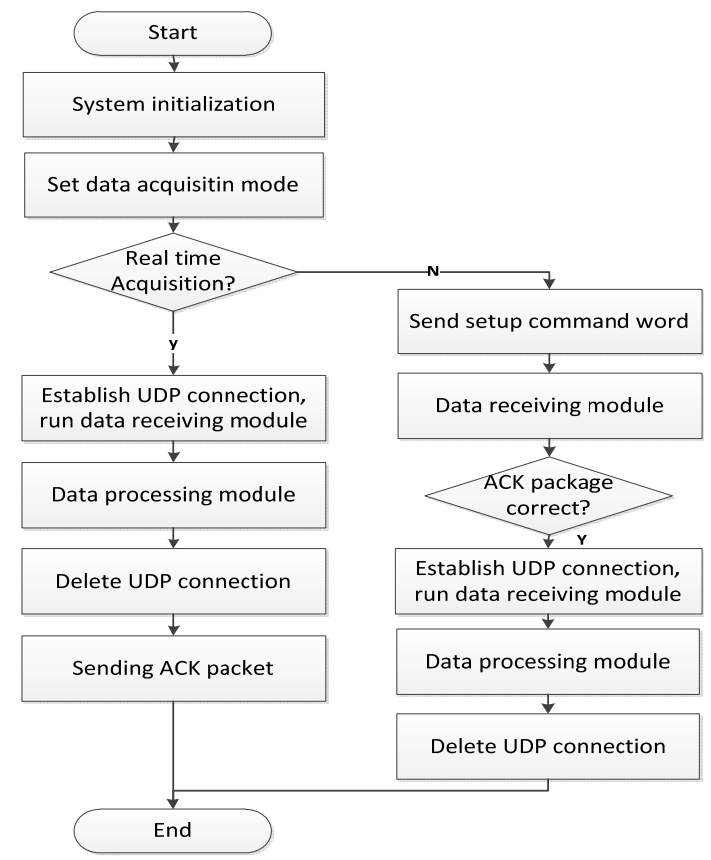

Figure 4. The flow diagram of data acquisition module

\section{B. The design of monitoring points}

The monitoring points use Linux as the software development platform. In order to make the whole system can work effectively and exert the advantage of the embedded system, the software design of monitoring points adopt the design scheme of the intranet. Multiple monitoring points constitute an intranet, which determines the final data transmission direction by configuring the route. The software module structure diagram of monitoring point is shown in Fig.5.

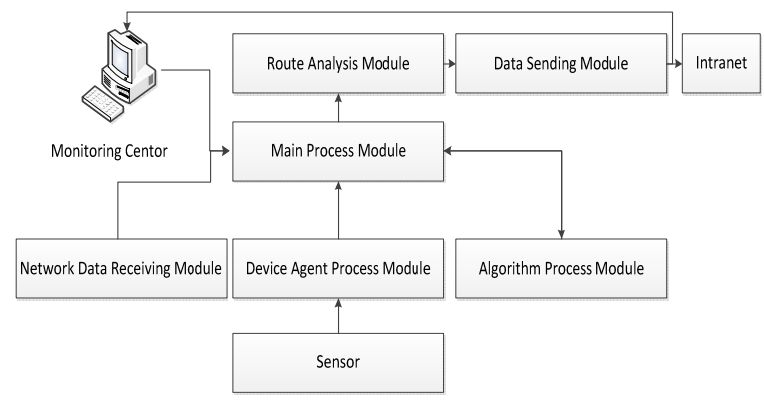

Figure 5. The software module structure diagram of monitoring point

Monitoring point is mainly responsible for collecting the data of each sensor ${ }^{[3]}$, after simple processing the data, sent to the destination, the destination may be another monitoring point or upper levels monitoring center, and also may be the monitoring point algorithm process. This design makes the whole remote structure monitoring system more intelligent. In the intranet constructed by multiple monitoring points, we can through the way of increasing or decreasing algorithm to increase or decrease intelligent task of each monitoring point ${ }^{[4]}$. Following the main process module of monitoring point will be introduced.

The main process is responsible for receiving three data packets, one is from the data packets of device agent process, one is from the network sock packets, and another is from sock local packets of algorithm process. After receiving the data packet, open the route analysis protocol, check route table, the packet of different sources will go to different destinations, finally open the sending process module, and send data packets.

\section{CONCLUSIONS}

The overall design, software module structure and realized mode of the remote structural monitoring system based on LABVIEW and Linux are proposed in this paper. The system has realized data packet no loss through the communication mechanism, its characteristic is the high stability and reliability. Interactive acquisition, analysis interface have developed in the Labview platform, and the real-time online monitoring and control have realized. In the Linux platform, make full use of the advantage of embedded system, using intranet hierarchical structure, to enable whole monitoring system more efficient, intelligent. The system can combine with a variety of sensor data acquisition system to realize the remote structural monitoring for different specific objects, and has wide application prospect in many monitoring fields.

\section{SUMMARY}

Yubing Jing, female, born in 1983 April, teacher of Zhonghuan Information College Tianjin University of Technology, master, the main research directions include intelligent control technology and remote detection system.Tel:13920326959, E-mail: jingyubing@163.com,

Address: No. 99, Liu Kou Road,Yangliuqing town, Xiqing District, Tianjin.

\section{REFERENCE}

[1] Hui Li, ZHOU Wen-song. Journal of intelligent monitoring system for large bridge structure [J]. Journal of civil engineering, 2006 (2), 47 52.

[2] LIU Jun-hua. Design of virtual instrument based on LabVIEW[M].Beijing: Electronic Industry Press, 1999, 255 257.

[3] Yang J N, Lei Y, Lin S, Huang N. Hibert huang Based Approach for Structural Damage Detection[J]. Journal of Engineering Mechanics, 2004, 130(I): 85 95.

[4] Shuping Liu, GaoFang. Construction of embedded Linux Operating System Based on The ARM Processor[C]// Proceedings of 2011 4th IEEE International Conference on Computer Science and Information Technology(ICCSIT 2011) VOL09,2011:83-87. 